\title{
Differences between two types of dual tasks according to the educational level in older adults
}

\author{
Pablo Tomas-Carus ${ }^{\mathrm{a}, \mathrm{b}, *}$, Hugo Rosado ${ }^{\mathrm{a}, \mathrm{b}}$, Catarina Pereira ${ }^{\mathrm{a}, \mathrm{b}}$, José Marmeleira ${ }^{\mathrm{a}, \mathrm{b}}$, Guida Veiga ${ }^{\mathrm{a}, \mathrm{b}}$, \\ Daniel Collado-Mateo ${ }^{\mathrm{c}}$ \\ ${ }^{a}$ Departamento de Desporto e Saúde, Escola de Ciências e Tecnologia, Universidade de Évora, Portugal \\ ${ }^{\mathrm{b}}$ Comprehensive Health Research Center (CHRC), University of Évora, Portugal \\ ${ }^{\text {c } C e n t r e ~ f o r ~ S p o r t ~ S t u d i e s, ~ R e y ~ J u a n ~ C a r l o s ~ U n i v e r s i t y, ~ 28943, ~ F u e n l a b r a d a, ~ M a d r i d, ~ S p a i n ~}$
}

\section{A R T I C L E I N F O}

\section{Keywords:}

Elderly

Simultaneous task

Timed up and go

Educational level

Falling risk

\begin{abstract}
A B S T R A C T
Introduction: For dual-task paradigms, the timed up and go (TUG) test along with other cognitive or motor tasks has been used to evaluate and predict the risk of falling in older adults. However, the interference between motor-cognitive tasks can differ by the cognitive task.

Objective: To evaluate the performance of the TUG test under a single task condition and two dual-task conditions in older adults and to explore the effect of educational level on task performance.

Methods: A total of 418 older adults (328 females) voluntarily participated in this study. The TUG test was administered as a single task and a dual task with one secondary simultaneous task: counting aloud backward from 100 or naming animals. Comparisons were performed to determine the interference caused by each cognitive task on the motor task, and correlation analysis was performed to explore the role of educational level. Results: The animal task led to a poorer TUG performance and a higher dual-task cost than did the counting task. Furthermore, the motor task led to a higher percentage of errors and cognitive stops in the animal task. Educational level plays a significant role in the interaction between tasks.

Conclusions: Between-task interference differs by the type of cognitive task performed and the educational level of the participants. The results of the present study should be considered when dual-task assessments are planned for older adults.
\end{abstract}

\section{Introduction}

In dual-task paradigms, two or more tasks are performed simultaneously and individuals' performance is assessed; when physical fitness is involved, dual-task paradigms allow a more physiological and realistic evaluation of individuals' ability to perform activities of daily living, which often involve simultaneous cognitive and/or physical activities (Yuan, Blumen, Verghese, \& Holtzer, 2015). For example, it is common for people to perform physical tasks such as walking or climbing stairs while thinking about other unrelated ideas or carrying something in their hands. However, when undergoing single-task physical fitness evaluations, participants are often required to focus all their attention on the execution of the test, which may influence the results of the test to a certain extent in terms of performance (Eggenberger, Tomovic, Munzer, \& de Bruin, 2017; Smith, Walsh, Doyle, Greene, \& Blake, 2017) or posture (Woollacott \& Shumway-Cook, 2002), as well as limit the generalizability of the results to the participants' daily lives
(McFadyen, Gagne, Cossette, \& Ouellet, 2017).

Dual-task paradigms have also been identified as good methods of evaluating predictors of the risk of falling (Ambrose, Paul, \& Hausdorff, 2013; Tomas-Carus et al., 2019), which is a major health problem among older adults (James et al., 2020). The assessment of the risk of falling is a complex task because there are multiple factors that can increase or decrease the results (Ambrose et al., 2013; Enderlin et al., 2015). Thus, simple methods of evaluating physical fitness may be limited. However, the predictive ability of such a simple assessment could be increased by adding a secondary cognitive task, enabling the simultaneous evaluation of cognitive and motor performance and the interference that exists between them. Regarding "between-task interference", Lacour, Bernard-Demanze, and Dumitrescu (2008)) suggested three different models for assessing postural and cognitive dual-task interactions. The first is the cross-domain competition model, which states that when two tasks are performed simultaneously, they compete for attention, and the result is a reduction in performance for both tasks.

\footnotetext{
* Corresponding author at: Department of Sport and Health, University of Évora, Romão Ramalho, 59, 7000-671, Évora, Portugal.

E-mail address: ptc@uevora.pt (P. Tomas-Carus).
} 
The second model is a U-shaped nonlinear interaction model and establishes that the performance in the motor task can improve or diminish depending on the attentional demands of the cognitive task. Thus, when both the motor and cognitive tasks in a dual-task paradigm are simple, an individual's performance in the primary task (e.g., postural control in balance tasks) can even increase, possibly due to automated processes. The third model is a task prioritization model, in which the relevance of safety increases when two different tasks require attention. Therefore, according to this last model, when older adults perceive that they are at risk of falling, they prioritize the motor task to avoid falling.

In addition to static balance tests, the timed up and go (TUG) test has been widely used to evaluate physical function and risk of falling in elderly people (Nightingale, Mitchell, \& Butterfield, 2019). This test has been recommended by the U.S. Centers for Disease Control and Prevention (CDC) to assess gait and balance and to detect the risk of falling among older adults. For dual-task paradigms, previous studies have used the TUG test along with other cognitive or motor tasks, such as reciting alternate letters of the alphabet (A-C-E, etc.) (Donoghue, Savva, Borsch-Supan, \& Kenny, 2019), counting backward in threes (Hofheinz \& Mibs, 2016), carrying a glass of water (Hofheinz \& Mibs, 2016), counting aloud backward from 100 (Asai et al., 2018), or providing answers to continuous arithmetic operations (Ponti, Bet, Oliveira, \& Castro, 2017). Given that interference is bidirectional and that the two tasks influence each other, performance in not only the motor task but also the secondary task must be assessed; for instance, the number of successful and wrong answers in the cognitive task can be counted (Ansai et al., 2017; Lima, Ansai, Andrade, \& Takahashi, 2015; Porciuncula, Rao, \& McIsaac, 2016).

To conduct an appropriate dual-task evaluation, the primary and secondary tasks selected should be adequate according to the motor and cognitive level of the participants. In this regard, factors such as cognitive impairment or educational level may be considered and may largely influence the interference between tasks. Although many published studies have focused on dual-task evaluations in older adults, there is no consensus on the most appropriate types of secondary tasks. Therefore, the current study aimed to explore individuals' performance in the TUG test in single- and dual-task paradigms and compare the interference caused by two types of secondary cognitive tasks: an arithmetic task in which the individual counts aloud backward from 100 and a semantic task in which the individual names animals.

\section{Methods}

\subsection{Participants}

Community-dwelling older adults living in the district of Évora (Portugal) participated in this cross-sectional study. Participants were recruited by invitation through verbal communication, posters, and flyers distributed in community settings, such as senior universities, city halls, and recreative/cultural centers. Epidemiologic statistical OpenEpi software (Sullivan, Dean, \& Soe, 2009) and the national census (INE, 2011) (adults $\geq 65$ years old) were used to estimate the minimum sample size necessary to represent the population of interest and provide statistically significant results. The sample size needed was 385 participants. The inclusion criteria were individuals who were a) male or female community-dwelling participants, b) aged $\geq 65$ years old, and c) living at home independently (a score of $\geq 18$ on the composite physical function scale) (Rikli \& Jones, 2013). Individuals were excluded if they were diagnosed with dementia, Parkinson's disease, or vertigo. A total of 513 participants were enrolled in the present study, but 95 were excluded on the basis of the inclusion criteria $(n=6$ criteria "a"; $n=24$ criteria " $b " ; n=31$ criteria "c") or exclusion criteria $(\mathrm{n}=34)$. Table 1 shows the main characteristics of the remaining 418 participants ( 90 males and 328 females). All participants provided their written informed consent in a pretest meeting. This study was approved
Table 1

Main characteristics of the sample according to sex.

\begin{tabular}{|c|c|c|c|c|c|}
\hline \multirow{3}{*}{$\begin{array}{l}\text { Fallers in the last } 6 \\
\text { months }\end{array}$} & \multirow{2}{*}{\multicolumn{2}{|c|}{$\frac{\text { Women }(\mathrm{n}=328)}{147(44.82 \%)}$}} & \multirow{2}{*}{\multicolumn{2}{|c|}{$\frac{\text { Men }(n=90)}{26(28.89 \%)}$}} & \multirow{3}{*}{$\begin{array}{l}\text { P-value } \\
0.007\end{array}$} \\
\hline & & & & & \\
\hline & Median & IQ range & Median & IQ range & \\
\hline Age (years) & 72 & 9 & 73 & 9 & 0.175 \\
\hline BMI $\left(\mathrm{kg} / \mathrm{m}^{2}\right)$ & 28.8 & 5.4 & 27.9 & 4.6 & 0.068 \\
\hline Educational level (years) & 4 & 2 & 4 & 3 & 0.058 \\
\hline Physical function (CPF) & 22 & 6 & 24 & 2 & $<0.001$ \\
\hline
\end{tabular}

BMI: Body Mass Index; IQ: interquartile; CPF: Composite Physical Function.

by the University of Évora Ethics Committee (reference number 16012) and was performed in accordance with the Declaration of Helsinki. Data were collected between April 2017 and January 2018.

\subsection{Procedure}

Assessments were performed individually by the same evaluator who held a degree in Sport Sciences. Sociodemographic evaluations and questionnaires/scales were administered in a quiet room, and physical fitness and body composition were assessed in a laboratory. The evaluator was blinded to the study objectives.

\subsubsection{Single-task timed up and go (TUG) test}

The TUG test was performed to assess gait and balance (Podsiadlo \& Richardson, 1991). A chair (approximately $43 \mathrm{~cm}$ tall) was placed against and facing a wall and at a distance of $2.44 \mathrm{~m}$ from a mark on the floor. After the command "Ready? Set, Go!", each participant had to stand up from the chair, walk and turn around at the mark as quickly as possible, and sit down again in the chair. After explaining the test protocol to the participant, the researcher performed a demonstration. Then, the participant executed one practice trial and two test trials. The time (s) it took for the participant to complete each test trial was measured.

Ten minutes after the single-task TUG test was performed, two dualtask TUG tests were performed: i) the counting backward timed up and go (TUG-C) test and ii) the naming animals timed up and go (TUG-A) test. Both dual-task TUG versions were performed in a random order, with another $10 \mathrm{~min}$ between each test.

\subsubsection{Counting backward timed up and go (TUG-C) test}

The TUG-C test was performed to assess the dual-task ability with a motor and an arithmetic task. The motor task followed the same procedure as the TUG test. Additionally, the cognitive task consisted of counting down by one from 100 while performing the motor task as quickly and safely as possible. After receiving an explanation and demonstration by the researcher, the participant executed one practice trial. Afterwards, the participant executed one test trial to minimize the learning effect. The test trial was recorded by video, and all TUG-C variables, namely, time to completion (s), number of cognitive stops, and number of errors, were calculated on the basis of the video recording. Moreover, one new variable was computed following the methods from a previous study (Tomas-Carus et al., 2019): "TUGC + Number of Cognitive Stops + Number of Errors". The dual-task cost (\%) was also calculated as $100 \mathrm{x}$ the difference between the single TUG and TUG-C values divided by the sum of the single TUG and TUG-C values (Asai et al., 2018).

\subsubsection{Naming animals timed up and go (TUG-A) test}

The TUG-A was performed to assess dual-task ability with a semantic task that was similar to that used in a previous study (Cedervall, Kilander, \& Aberg, 2012). The motor task followed the same procedures as the TUG test. Additionally, the cognitive task consisted of naming as 
many different animals as possible while performing the motor task as quickly and safely as possible. After providing instructions, the researcher performed a demonstration starting from the sitting position in the chair. Then, he/she started naming different animals, and when he or she named the second animal, the researcher stood up from the chair, walked to and turned around at the mark as quickly as possible, and continued naming animals (maximum up to 5) until he or she sat down again. The participants were encouraged to choose animals different from those mentioned by the researcher, as well as animals from different species without repetitions. The participants executed only one test trial to minimize the learning effect. The test was recorded by video, and all TUG-A variables, namely, time to completion (s), number of cognitive stops, and number of errors, were calculated on the basis of the video recording. The dual-task cost and the new variable "TUGC + Number of Cognitive Stops + Number of Errors" were also calculated.

\subsubsection{Fall history and education level}

Fall history was assessed by an interviewer. A fall was defined as "an unexpected event in which the participant came to a rest on the ground, floor, or a low surface" (Lamb, Jorstad-Stein, Hauer, \& Becker, 2005). Information on the fall (e.g., whether there were fall-related injuries, the fall occurred indoors or outdoors, or the fall was accidental) was also collected, but it was not used in this manuscript. A faller was considered a participant who had experienced at least one fall in the previous 6 months. Educational level was assessed by the question "How many years of schooling have you completed?" in the questionnaire on sociodemographic characteristics (age, sex, marital status, and health conditions).

\subsubsection{Physical function and body composition}

Physical function was assessed by the 12-item composite physical function (CPF) scale (Rikli \& Jones, 2013), which measures the physical independence level and the range of functional abilities. The CPF score ranges from 0 (worst) to 24 (best) points. On the basis of the scores, the participants were considered to have "high function" (24 points), "moderate function" (18-23 points), or low function ( $<18$ points). Body weight $(\mathrm{kg})$ and height $(\mathrm{m})$ were measured without shoes with a stadiometer (Seca 206, Hamburg, Germany) and an electronic scale (Seca 760, Hamburg, Germany), and body mass index (BMI) was calculated using a formula $\left(\mathrm{kg} / \mathrm{m}^{2}\right)$.

\subsection{Statistical analysis}

Nonparametric statistical analyses were conducted on the basis of the results of the Kolmogorov-Smirnov test. First, the main characteristics of the men and women were compared. For continuous variables, the Mann-Whitney $U$ test was used, while the chi-squared test was used to examine the difference in the percentages of male and female fallers. To understand the role of educational level in the dual-task paradigm, two subgroups were created based on the median number of years: 4 . Thus, the study population was divided into those with 4 or fewer years of schooling ( $\leq 4$ years) and those with more than 4 years ( $>4$ years). The differences between these two groups were evaluated using the Mann-Whitney $U$ test. Statistical power calculations were performed for this analysis considering the fact that the study population was divided into 4 groups: 1 ) women with a low educational level ( $\leq 4$ years), 2 ) men with a low educational level ( $\leq 4$ years), 3 ) women with a high educational level ( $>4$ years) and 4 ) men with a high educational level ( $>4$ years). For the single-task TUG test, the statistical power calculations revealed that differences between the two women groups (based on the educational level) could be detected with a power of $99 \%$ and an alpha value of 0.05 when the groups included 225 and 103 women. On the other hand, in the case of men, the statistical power was $86.4 \%$ when the groups included 51 and 39 men. Repeated measures ANOVA was performed to compare individuals' performance in the TUG-C test and the TUG-A. These analyses were conducted with Bonferroni adjustments and with consideration of the participants' sex and educational level. Finally, nonparametric bivariate correlations were conducted to determine the associations of TUG variables with educational level and the number of falls. Given the between-sex differences in TUG performance, this correlation analysis were conducted for the two sexes separately.

\section{Results}

The main characteristics of the participants are reported in Table 1. There was a significantly higher prevalence of falls among the women than among the men ( $44.82 \%$ versus $28.89 \%$, respectively). Overall, the median number of years in school was 4 in both the men and women, but the mean was slightly higher in the men (5.96 years) than in the women (5.55 years). Furthermore, significant differences between men and women were also observed in the CPF scale score. The high median CPF score for the men indicates that they have high or advanced functionality in general and are able to perform the 12 tasks without any help. On the other hand, the median score for functionality of the women was 22 , which can be classified as "moderate".

To assess the differences between those with low educational levels ( $\leq 4$ years) and those with 5 or more years of schooling, an independent samples test was conducted (see Fig. 1). The results showed that there was a significant difference in all versions of the TUG test. Specifically, in the single-task TUG test, both the women (mean $6.44 \mathrm{~s} \pm 1.34 \mathrm{~s} v \mathrm{~s}$ $7.35 \mathrm{~s} \pm 2.22 \mathrm{~s}$ ) and men (mean $6.06 \mathrm{~s} \pm 1.52 \mathrm{~s} v s 7.51 \mathrm{~s} \pm 2.86 \mathrm{~s}$ ) with a higher educational level had better performance. These differences were also observed in the dual-task TUG test. For the TUG-A test, the women with a high educational level ( $>4$ years) had a mean completion time of $10.11 \mathrm{~s} \pm 3.40 \mathrm{~s}$, and those with a low educational level had a mean completion time of $11.71 \mathrm{~s} \pm 4.69 \mathrm{~s}$; in contrast, among men, the time required by those with low educational level was 22.65 $\%$ higher than the time required by those with more than 4 years of schooling. Regarding the TUG-C test, the women with a low educational level needed more than $20 \%$ more time than did those with $>4$ years of schooling, while for the men, the mean time of those with a low educational level was more than $30 \%$ higher than that of those with a high educational level. All differences were significant.

Table 2 summarizes the differences between the two different dual tasks selected. Regarding the interference caused by the cognitive tasks in all men and women, the animal task (TUG-A) required the participants to take more time to complete the TUG and led to a higher dualtask cost. Furthermore, the physical task also led to a higher percentage of errors and a higher number of cognitive stops in the animal task. However, when educational level was considered, those relationships were no longer significant. In this regard, men with low educational levels showed a similar percentage of errors for both tasks and similar times to complete the TUG-A and TUG-C tasks. Additionally, nonsignificant differences in the dual-task cost between the two cognitive tasks were found.

The correlations between the number of years of education and the number of falls in the last 6 months and the TUG variables were assessed to identify disparities in the two dual-task procedures selected (Table 3). In both men and women, there was a significant association between educational level and all variables of the single and dual-task TUG test that were assessed ( $\mathrm{p}<0.05$ ), except for the dual-task cost caused by the secondary task of naming animals (TUG-A). Regarding the participants' history of falls, a significant correlation was observed between the number of falls in the last 6 months and all single and dual TUG test times among men, while only the single TUG and TUG-C test times were significantly related to falls among women. Regarding dualtask cost, only the TUG-C test was significantly associated with falls in women. 

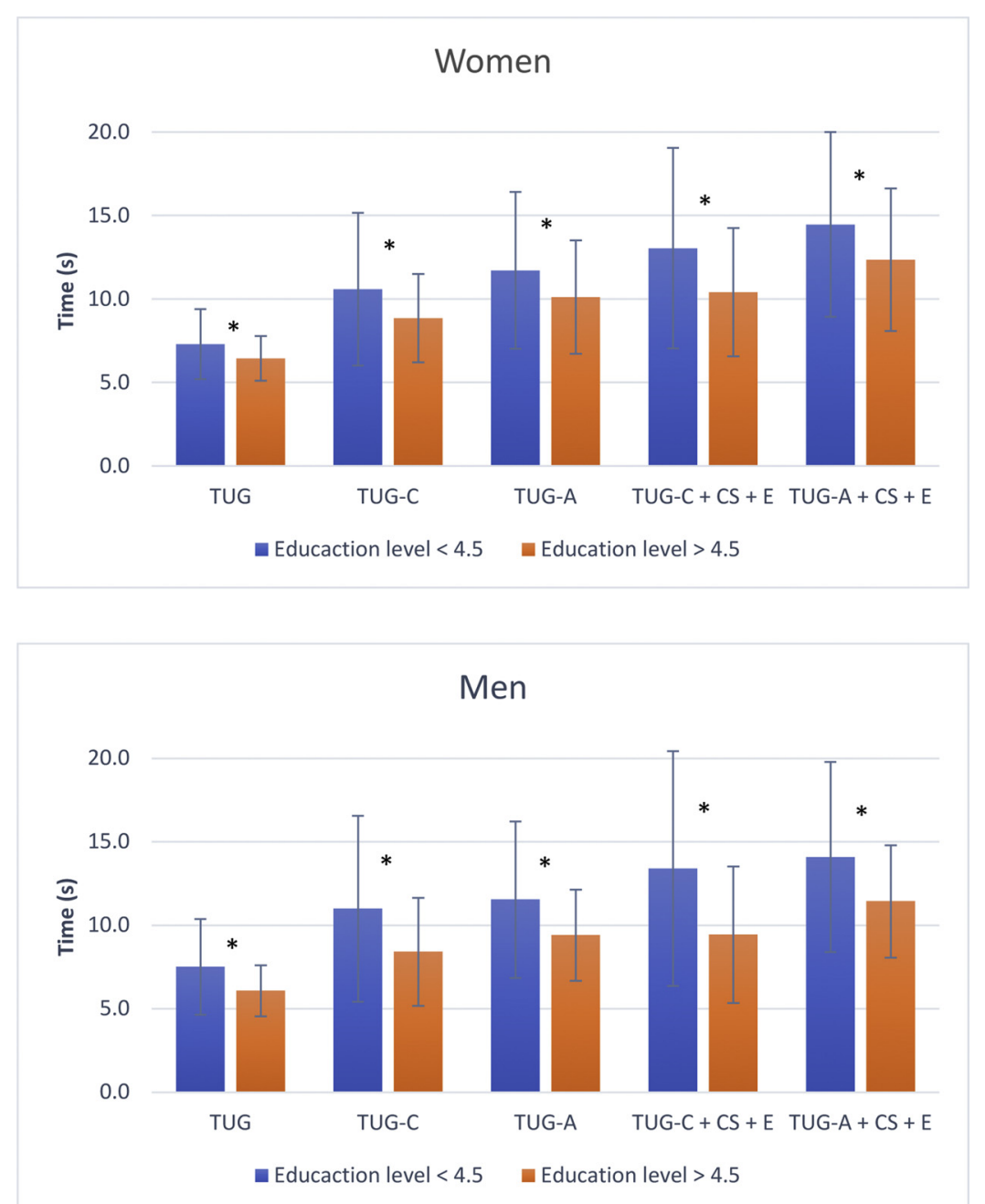

Fig. 1. Comparison between TUG types according to educational level in women and men. * Significant differences between those with $<4.5$ ( 4 years or less) and those with $>4.5$ years (more than 4 years) of formal education. TUG: Timed-up and go test; TUG-C: TUG counting backwards; TUG-A: TUG naming animals; TUGC + CS + E: TUG-C adding the number of cognitive stops and the number of errors; TUG-A + CS + E: TUG-A adding the number of cognitive stops and the number of errors.

\section{Discussion}

The current article aimed to evaluate the differences between two different dual-task tests according to the participants' sex and educational level. The main finding was that the two secondary tasks selected led to significantly different interferences in the motor task and that the motor task had a distinct interference with the animal task (TUG-A) and with the counting task (TUG-C). Furthermore, educational level was shown to be a relevant variable that should be considered when dualtask evaluations are planned. The results showed that the single and dual-task variables had significantly lower values in the participants with a low educational level than in the participants with $>4$ years of schooling. This last finding is in line with that of previous research showing that a higher level of education is associated with better performance in the dual-task TUG test (Gomes Gde et al., 2015). This may be explained by the higher cognitive reserve observed in those with a higher educational level, and they may therefore be able to maintain higher levels of brain functions over time (Stern, 2012). In this regard, a previous study showed that reduced performance in the TUG-A test may be related to neurodegeneration, as assessed by Alzheimer's disease cerebrospinal fluid biomarkers (Ahman et al., 2019). Thus, according to our results and to previous research, educational level seems to influence the between-task interference, which highlights the importance of selecting and adapting cognitive and/or motor tasks in accordance with the participant's educational level. Future studies should explore the adaptation of tasks based on this variable, such as varying the level of complexity of cognitive tasks (for instance, counting backward in twos, in threes, counting forward, etc.). Furthermore, other tasks, such as texting on a smartphone, could be considered, given its notable presence in daily life and the potential hazards associated with texting when simultaneously performing another task (Hsiao, Belur, Myers, Earhart, \& Rawson, 2020).

Task selection is of paramount importance regarding assessments planned for elderly people. Some tasks may be too difficult, while others may be too simple to detect a specific interference. This issue is exacerbated in a largely heterogeneous population such as older adults, so researchers may need to divide participants into subgroups to elicit comparable responses when evaluating responses to the dual-task paradigm. As mentioned in the introduction section, Lacour et al. (2008) suggested three different models for assessing interactions and interference between tasks. Those interactions are conditioned by the characteristics of the tasks (for instance, the perceived risk of falling in a motor task) and the attentional demands. This is conditioned, in turn, by the difficulty of the task and the ability of the participant to complete it. In the current study, we observed that the animal task (TUG-A) led to higher interferences in the motor task than in the counting task (TUG-C) and that interference was mutual, leading to a larger percentage of errors in the animal task than in the counting task. Interestingly, 
Table 2

Comparison between the two types of dual task TUG according to the educational level and sex.

\begin{tabular}{|c|c|c|c|c|c|c|c|c|c|c|c|c|}
\hline & \multicolumn{6}{|c|}{ Women } & \multicolumn{6}{|l|}{ Men } \\
\hline & \multicolumn{2}{|c|}{$\begin{array}{l}\text { Education level } \\
\leq 4\end{array}$} & \multicolumn{2}{|c|}{ Education level $>4$} & \multicolumn{2}{|l|}{ Total } & \multicolumn{2}{|c|}{$\begin{array}{l}\text { Education level } \\
\leq 4\end{array}$} & \multicolumn{2}{|c|}{$\begin{array}{l}\text { Education level } \\
>4\end{array}$} & \multicolumn{2}{|l|}{ Total } \\
\hline & Mean & SD & Mean & SD & Mean & $\mathrm{SD}$ & Mean & SD & Mean & SD & Mean & SD \\
\hline $\begin{array}{r}\text { TUG-C } \\
(\mathrm{s})\end{array}$ & 10.59 & 4.57 & 8.85 & 2.65 & 10.04 & 4.14 & 10.99 & 5.57 & 8.41 & 3.24 & 9.87 & 4.85 \\
\hline $\begin{array}{r}\text { TUG-A } \\
\text { (s) }\end{array}$ & 11.71 & 4.69 & 10.11 & 3.40 & 11.21 & 4.38 & 11.54 & 4.68 & 9.41 & 2.73 & 10.62 & 4.08 \\
\hline $\mathrm{p}$-value & \multicolumn{2}{|c|}{$<0.001$} & \multicolumn{2}{|c|}{$<0.001$} & \multicolumn{2}{|c|}{$<0.001$} & \multicolumn{2}{|l|}{0.233} & \multicolumn{2}{|l|}{0.003} & \multicolumn{2}{|l|}{0.012} \\
\hline Hedges's g (95 \% CI) & \multicolumn{2}{|c|}{$0.24(0.14$ to 0.34$)$} & \multicolumn{2}{|c|}{$0.41(0.23$ to 0.60$)$} & \multicolumn{2}{|c|}{0.27 (0.19 to 0.36$)$} & \multicolumn{2}{|c|}{$0.10(-0.07$ to 0.28$)$} & \multicolumn{2}{|c|}{0.33 (0.11 to 0.55$)$} & \multicolumn{2}{|c|}{$0.17(0.03$ to 0.30$)$} \\
\hline \%Errors TUG-C & 16.99 & 21.48 & 8.36 & 12.98 & 14.27 & 19.59 & 14.43 & 23.24 & 4.35 & 7.94 & 10.02 & 18.80 \\
\hline \% Errors TUG-A & 20.61 & 24.51 & 11.03 & 18.58 & 17.59 & 23.20 & 16.71 & 27.28 & 16.64 & 20.90 & 16.68 & 24.55 \\
\hline p-value & \multicolumn{2}{|l|}{0.068} & \multicolumn{2}{|l|}{0.226} & \multicolumn{2}{|l|}{0.029} & \multicolumn{2}{|l|}{0.602} & \multicolumn{2}{|l|}{0.002} & \multicolumn{2}{|l|}{0.027} \\
\hline Hedges's g (95 \% CI) & \multicolumn{2}{|c|}{$0.15(-0.01$ to 0.33$)$} & \multicolumn{2}{|c|}{$0.16(-0.10$ to 0.43$)$} & \multicolumn{2}{|c|}{0.15 (0.02 to 0.29$)$} & \multicolumn{2}{|c|}{$0.09(-0.24$ to 0.42$)$} & \multicolumn{2}{|c|}{$0.76(0.28-1.26)$} & \multicolumn{2}{|c|}{$0.30(0.04$ to 0.57$)$} \\
\hline Cognitive Stops TUG-C & 0.92 & 0.88 & 0.63 & 0.79 & 0.83 & 0.87 & 0.75 & 0.71 & 0.440 & 0.72 & 0.61 & 0.73 \\
\hline Cognitive stops TUG-A & 1.60 & 0.90 & 1.57 & 1.04 & 1.59 & 0.95 & 1.47 & 0.98 & 1.080 & 0.90 & 1.30 & 0.97 \\
\hline $\mathrm{p}$-value & \multicolumn{2}{|c|}{$<0.001$} & $<0.0$ & & $<0.00$ & & $<0.0$ & & $<0.0$ & & $<0.0$ & \\
\hline Hedges's g (95 \% CI) & $0.76(0$ & to 0.93 ) & 1.00 & 1.29) & $0.84(0$ & to 0.98 ) & $0.83(0$ & -1.27) & $0.77(0$ & $-1.15)$ & 0.80 & $-1.09)$ \\
\hline Dual task cost TUG-C (\%) & 32.45 & 22.44 & 29.33 & 19.70 & 31.47 & 21.63 & 34.45 & 17.55 & 28.76 & 19.63 & 31.98 & 18.59 \\
\hline Dual task cost TUG-A (\%) & 42.54 & 23.43 & 40.91 & 22.25 & 42.03 & 23.04 & 40.01 & 23.03 & 41.59 & 16.58 & 40.7 & 20.4 \\
\hline p-value & $<0.0$ & & $<0.0$ & & $<0.00$ & & 0.066 & & $<0.0$ & & $<0.0$ & \\
\hline Hedges's g $(95 \% \mathrm{CI})$ & $0.44(0$ & to 0.58 ) & 0.54 & o 0.75$)$ & $0.27(0$ & to 0.41 ) & $0.27(-$ & to 0.56 ) & $0.69(0$ & $-1.06)$ & 0.44 & to 0.67 ) \\
\hline
\end{tabular}

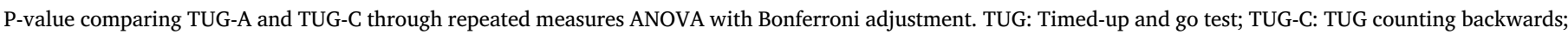
TUG-A: TUG naming animals. SD: standard deviation.

the largest between-task difference in the percentage of errors was observed for men with $>4$ years of education. This subgroup had almost a four times higher percentage of errors in the animal task $(4.3 \pm 7.94)$ than in the counting task $(16.64 \pm 20.90)$. This finding, along with previous findings on interference in the motor task, might suggest that the counting task is too simple for those with higher educational levels, therefore demanding less attention. However, in the subgroup comprised of women with $>4$ years of education, a difference in the interference in the cognitive task (percentage of errors) was not observed, so no strong conclusions can be made. More research is therefore needed, as there are still some inconsistencies and questions on the interference of different tasks that need to be answered. For example, a recent study showed that age, sex and educational level do not influence an individual's ability to be attentive when performing two cognitive tasks simultaneously in healthy adults, including older adults (Sebastian \& Mediavilla, 2017). Furthermore, motivational and volitional aspects should also be considered since one secondary task may be considered engaging, challenging, or boring, depending on many variables such as the participant's sex or educational level, as well as other uncontrolled variables, such as previous experiences and personal preferences. Therefore, the motor or cognitive task selected for each group or population may affect the results and must be explored in future research.

The current study also showed that the dual-task cost caused by the counting task (TUG-C) was related to the educational level, so the higher the educational level was, the lesser the interference caused by that cognitive task on the motor task. Interestingly, this relation was not observed in the animal task (TUG-A), which means that educational level is related to the interference caused by the counting task but not with that caused by the animal task. Possibly, in a population comprised of people with substantial age-related heterogeneity, the animal task would be recommended. Regarding falls, the TUG-C task may be more related to the participants' history of falls than is the TUG-A task. However, most of the observed correlations between any TUG variable and falls were low, which could be related to the heterogeneity of the study population. It is known that there is a relationship between the TUG test and fall risk, but it has also been suggested that the time needed to complete the TUG test may not be a suitable tool for identifying individuals at high risk of falls (Barry, Galvin, Keogh, Horgan, \& Fahey, 2014; Vaillant et al., 2006). However, the addition of a secondary task such as counting backward may increase the ability to discriminate between fallers and nonfallers (Tomas-Carus et al., 2019).

The current study provides some useful information for selecting tasks on the basis of the characteristics of participants; however, there

Table 3

Correlations between TUG variables and both the educational level and the number of falls.

\begin{tabular}{|c|c|c|c|c|c|c|c|c|}
\hline & \multicolumn{4}{|l|}{ Women } & \multicolumn{4}{|l|}{ Men } \\
\hline & \multicolumn{2}{|l|}{ Educational level (years) } & \multicolumn{2}{|c|}{ Number of falls in the last 6 months } & \multicolumn{2}{|l|}{ Educational level (years) } & \multicolumn{2}{|c|}{ Number of falls in the last 6 months } \\
\hline & Correlation Coefficient & P-value & Correlation Coefficient & P-value & Correlation Coefficient & P-value & Correlation Coefficient & P-value \\
\hline TUG & -.311 & $<0.001$ & .118 & 0.033 & -.365 & $<0.001$ & .426 & $<0.001$ \\
\hline TUG-C & -.315 & $<0.001$ & .132 & 0.017 & -.426 & $<0.001$ & .410 & $<0.001$ \\
\hline TUG-A & -.249 & $<0.001$ & .093 & 0.095 & -.247 & 0.019 & .326 & 0.002 \\
\hline TUG-C + CS + E & -.350 & $<0.001$ & .101 & 0.069 & -.451 & $<0.001$ & .361 & $<0.001$ \\
\hline TUG-A + CS + E & -.258 & $<0.001$ & .106 & 0.056 & -.256 & 0.015 & .293 & 0.005 \\
\hline Dual task cost TUG-C & -.133 & 0.016 & .110 & 0.047 & -.267 & 0.011 & .072 & 0.502 \\
\hline Dual task cost TUG-A & -.032 & 0.559 & .032 & 0.562 & .109 & 0.305 & .065 & 0.545 \\
\hline
\end{tabular}

P-value of the Spearman's correlation test. TUG: Timed-up and go test; TUG-C: TUG counting backwards; TUG-A: TUG naming animals; TUG-C + CS + E: TUG-C adding the number of cognitive stops and the number of errors; TUG-A + CS + E: TUG-A adding the number of cognitive stops and the number of errors. 
are some limitations that should be mentioned. First, as mentioned above, the limited sample size, especially the smaller number of male participants than female participants prevented the division of the participants into age subgroups. The second limitation is related to the cross-sectional design, which limits the conclusions that can be made and the amount of information that can be used to assess falls; the participants' history of falls rather than the number of falls that occurred after the assessment was analyzed. Furthermore, the reliability and validity of the TUG-C and TUG-A should be evaluated in future research.

\section{Conclusion}

In the dual-task, there were relevant differences between the interference caused by the animal task and that caused by the counting task on the motor task/TUG performance. The animal task led to poorer TUG test performance, and the percentage of errors in that cognitive task was also larger than that in the counting task. Furthermore, educational level seems to play an important role in the interaction between motor-cognitive tasks, so it should be considered when dual-task assessments are selected. In this regard, the counting dual-task cost was significantly associated with the educational level, while the animal dual-task cost was not. Thus, the animal task might be recommended when participants have a heterogeneous level of education.

\section{Funding}

This study is part of a larger project entitled "Ageing Safely in Alentejo. Preventing Falls and Violence against the Elderly. Understanding for Action - (ESACA)" and was supported by the European Fund for regional development (FEDER) allocated by European Union through the Horizon 2020 ProgramALT20-03-0145FEDER-000007. The funder played no role in the study design, the data collection and analysis, the decision to publish, or the preparation of the manuscript.

\section{Authors' contributions}

Pablo Tomas-Carus: conceptualization, methodology, writing-original draft preparation; Hugo Rosado: data collection, writing-reviewing; Catarina Pereira: writing-reviewing; José Marmeleira: writingreviewing; Guida Veiga: writing-reviewing; Daniel Collado-Mateo: methodology, writing-original draft preparation. All authors read and approved the final manuscript.

\section{Declaration of Competing Interest}

The authors declare that they have no conflict of interests.

\section{References}

Ahman, H. B., Giedraitis, V., Cedervall, Y., Lennhed, B., Berglund, L., McKee, K., \& Aberg, A. C. (2019). Dual-task performance and neurodegeneration: correlations between timed up-and-go dual-task test outcomes and Alzheimer's disease cerebrospinal fluid biomarkers. Journal of Alzheimer's Disease : JAD, 71(s1), S75-s83. https://doi.org/10. 3233/JAD-181265.

Ambrose, A. F., Paul, G., \& Hausdorff, J. M. (2013). Risk factors for falls among older adults: A review of the literature. Maturitas, 75(1), 51-61. https://doi.org/10.1016/j. maturitas.2013.02.009.

Ansai, J. H., Andrade, L. P., Rossi, P. G., Takahashi, A. C. M., Vale, F. A. C., \& Rebelatto, J. R. (2017). Gait, dual task and history of falls in elderly with preserved cognition, mild cognitive impairment, and mild Alzheimer's disease. Brazilian Journal of Physical Therapy, 21(2), 144-151. https://doi.org/10.1016/j.bjpt.2017.03.010.

Asai, T., Oshima, K., Fukumoto, Y., Yonezawa, Y., Matsuo, A., \& Misu, S. (2018). Association of fall history with the timed up and go test score and the dual task cost: A cross-sectional study among independent community-dwelling older adults. Geriatrics \& Gerontology International, 18(8), 1189-1193. https://doi.org/10.1111/ggi.13439.

Barry, E., Galvin, R., Keogh, C., Horgan, F., \& Fahey, T. (2014). Is the timed up and go test a useful predictor of risk of falls in community dwelling older adults: A systematic review and meta-analysis. BMC Geriatrics, 14, 14. https://doi.org/10.1186/1471.
2318-14-14.

Cedervall, Y., Kilander, L., \& Aberg, A. C. (2012). Declining physical capacity but maintained aerobic activity in early Alzheimer's disease. American Journal of Alzheimer's Disease and Other Dementias, 27(3), 180-187. https://doi.org/10.1177/ 1533317512442996.

Donoghue, O. A., Savva, G. M., Borsch-Supan, A., \& Kenny, R. A. (2019). Reliability, measurement error and minimum detectable change in mobility measures: A cohort study of community-dwelling adults aged 50 years and over in Ireland. BMJ Open, 9(11), Article e030475. https://doi.org/10.1136/bmjopen-2019-030475.

Eggenberger, P., Tomovic, S., Munzer, T., \& de Bruin, E. D. (2017). Older adults must hurry at pedestrian lights! A cross-sectional analysis of preferred and fast walking speed under single- and dual-task conditions. PloS One, 12(7), Article e0182180. https://doi.org/10.1371/journal.pone.0182180.

Enderlin, C., Rooker, J., Ball, S., Hippensteel, D., Alderman, J., Fisher, S. J., \& Jordan, K. (2015). Summary of factors contributing to falls in older adults and nursing implications. Geriatric Nursing (New York, NY), 36(5), 397-406. https://doi.org/10. 1016/j.gerinurse.2015.08.006.

Gomes Gde, C., Teixeira-Salmela, L. F., Fonseca, B. E., Freitas, F. A., Fonseca, M. L., Pacheco, B. D., \& Caramelli, P. (2015). Age and education influence the performance of elderly women on the dual-task timed up and go test. Arquivos de Neuro-psiquiatria, 73(3), 187-193. https://doi.org/10.1590/0004-282x20140233.

Hofheinz, M., \& Mibs, M. (2016). The prognostic validity of the timed up and go test with a dual task for predicting the risk of falls in the elderly. Gerontology \& Geriatric Medicine, 2, Article 2333721416637798. https://doi.org/10.1177/ 2333721416637798.

Hsiao, D., Belur, P., Myers, P. S., Earhart, G. M., \& Rawson, K. S. (2020). The impact of age, surface characteristics, and dual-tasking on postural sway. Archives of Gerontology and Geriatrics, 87, Article 103973. https://doi.org/10.1016/j.archger. 2019.103973.

INE (2011). Resident population (No.) by place of residence, sex and age group (by life cycles). 2020, fromhttp://www.ine.pt.

James, S. L., Lucchesi, L. R., Bisignano, C., Castle, C. D., Dingels, Z. V., Fox, J. T., \& Murray, C. J. L. (2020). The global burden of falls: Global, regional and national estimates of morbidity and mortality from the Global Burden of Disease Study 2017. Injury Prevention : Journal of the International Society for Child and Adolescent Injury Prevention. https://doi.org/10.1136/injuryprev-2019-043286.

Lacour, M., Bernard-Demanze, L., \& Dumitrescu, M. (2008). Posture control, aging, and attention resources: Models and posture-analysis methods. Neurophysiologie Clinique = Clinical Neurophysiology, 38(6), 411-421. https://doi.org/10.1016/j.neucli.2008. 09.005.

Lamb, S. E., Jorstad-Stein, E. C., Hauer, K., \& Becker, C. (2005). Development of a common outcome data set for fall injury prevention trials: The Prevention of Falls Network Europe consensus. Journal of the American Geriatrics Society, 53(9), 1618-1622. https://doi.org/10.1111/j.1532-5415.2005.53455.x.

Lima, L. C. A., Ansai, J. H., Andrade, L. P., \& Takahashi, A. C. M. (2015). The relationship between dual-task and cognitive performance among elderly participants who exercise regularly. Brazilian Journal of Physical Therapy, 19(2), 159-166. https://doi. org/10.1590/bjpt-rbf.2014.0082.

McFadyen, B. J., Gagne, M. E., Cossette, I., \& Ouellet, M. C. (2017). Using dual task walking as an aid to assess executive dysfunction ecologically in neurological populations: A narrative review. Neuropsychological Rehabilitation, 27(5), 722-743. https://doi.org/10.1080/09602011.2015.1100125.

Nightingale, C. J., Mitchell, S. N., \& Butterfield, S. A. (2019). Validation of the timed up and go test for assessing balance variables in adults aged 65 and older. Journal of Aging and Physical Activity, 27(2), 230-233. https://doi.org/10.1123/japa.20180049 .

Podsiadlo, D., \& Richardson, S. (1991). The timed "Up \& Go": A test of basic functional mobility for frail elderly persons. Journal of the American Geriatrics Society, 39(2), $142-148$.

Ponti, M., Bet, P., Oliveira, C. L., \& Castro, P. C. (2017). Better than counting seconds: Identifying fallers among healthy elderly using fusion of accelerometer features and dual-task timed up and go. PloS One, 12(4), Article e0175559. https://doi.org/10. 1371/journal.pone.0175559.

Porciuncula, F. S., Rao, A. K., \& McIsaac, T. L. (2016). Aging-related decrements during specific phases of the dual-task timed Up-and-Go test. Aging Clinical and Experimental Research, 28, 121-130. https://doi.org/10.1007/s40520-015-0372-x.

Rikli, R. E., \& Jones, C. J. (2013). Development and validation of criterion-referenced clinically relevant fitness standards for maintaining physical independence in later years. Gerontologist, 53(2), 255-267. https://doi.org/10.1093/geront/gns071.

Sebastian, M. V., \& Mediavilla, R. (2017). Does dual-task coordination performance decline in later life? Psicothema, 29(2), 223-228. https://doi.org/10.7334/ psicothema2016.274.

Smith, E., Walsh, L., Doyle, J., Greene, B., \& Blake, C. (2017). Effect of a dual task on quantitative timed up and go performance in community-dwelling older adults: A preliminary study. Geriatrics \& Gerontology International, 17(8), 1176-1182. https:// doi.org/10.1111/ggi.12845.

Stern, Y. (2012). Cognitive reserve in ageing and Alzheimer's disease. The Lancet Neurology, 11(11), 1006-1012. https://doi.org/10.1016/s1474-4422(12)70191-6.

Sullivan, K. M., Dean, A., \& Soe, M. M. (2009). OpenEpi: A web-based epidemiologic and statistical calculator for public health. Public Health Reports (Washington, DC : 1974), 124(3), 471-474. https://doi.org/10.1177/003335490912400320.

Tomas-Carus, P., Biehl-Printes, C., Pereira, C., Veiga, G., Costa, A., \& Collado-Mateo, D. (2019). Dual task performance and history of falls in community-dwelling older adults. Experimental Gerontology, 120, 35-39. https://doi.org/10.1016/j.exger.2019. 02.015 .

Vaillant, J., Martigne, P., Vuillerme, N., Caillat-Miousse, J. L., Parisot, J., Juvin, R., ... 
Nougier, V. (2006). [Prediction of falls with performance on timed "Up-and-Go" and one-leg-balance tests and additional cognitive tasks]. Annales de Readaptation et de Medecine Physique : Revue Scientifique de La Societe Francaise de Reeducation

Fonctionnelle de Readaptation et de Medecine Physique, 49(1), 1-7. https://doi.org/10. 1016/j.annrmp.2005.07.002.

Woollacott, M., \& Shumway-Cook, A. (2002). Attention and the control of posture and gait: A review of an emerging area of research. Gait \& Posture, 16(1), 1-14. https:// doi.org/10.1016/s0966-6362(01)00156-4.

Yuan, J., Blumen, H. M., Verghese, J., \& Holtzer, R. (2015). Functional connectivity associated with gait velocity during walking and walking-while-talking in aging: A resting-state fMRI study. Human Brain Mapping, 36(4), 1484-1493. 